\title{
Generation Times of Bacteria
}

\author{
By P. R. PAINTER \\ Department of Biology, University of California, Riverside, California 92502, U.S.A.
}

(Received 3 September 1974; revised 18 February 1975)

SUMMARY

Random delays in cell division lead to correlations between the generation times of mothers and their daughters and to a difference between the 'real' and the 'artificial' distributions of generation times. At present there is no satisfactory relation between the two distributions although both are useful in the analysis of growth. In a special case, it is shown that they are similar as long as the coefficient of variation of generation times is small.

\section{INTRODUCTION}

Powell (1964, 1969) distinguished between 'real' and 'artificial' distributions of generation times of bacteria: the 'real' distribution of generation time $\tau$, with frequency function here denoted $\mathscr{P}(\tau)$, is defined on a sample of newly formed cells in an exponentially growing population; whereas the 'artificial' distribution is defined on one or more lines of descent in an exponentially growing population. A line of descent is defined by randomly selecting one of each pair of daughter cells and removing it and all of its descendants from consideration. The remaining cells comprise the sample where $f(\tau)$, the 'artificial' frequency function of generation times, is defined. Mathematical analysis of $f(\tau)$ is usually easier than that of $\mathscr{P}(\tau)$ since $f(\tau)$ can be interpreted as the frequency function of waiting times in a stationary stochastic process, and such processes are usually much easier to analyse than are branching processes such as bacterial growth. Thus a relationship between $f(\tau)$ and $\mathscr{P}(\tau)$ is needed so that conclusions derived from analysis of the 'artificial' distribution can be transformed to apply to the 'real' distribution. Moreover, it is possible to measure either $f(\tau)$ or $\mathscr{P}(\tau)$, so that a transformation might be a useful means of comparing experimental results.

Powell (1969) points out that, in the absence of correlations between generation times, $f(\tau)$ and $\mathscr{P}(\tau)$ are identical. To analyse cases where generation times are dependent, Powell (1964, I969) introduces the joint frequency function $h(\xi, \tau)$ of the generation times $\xi$ of the mother and $\tau$ of the daughter in the sample where $f(\tau)$ is defined. Similarly, $\mathscr{H}(\xi, \tau)$ denotes the joint frequency of the generation times of mothers and their daughters defined on a sample of dividing mothers in the population where $\mathscr{P}(\tau)$ is defined. Powell asserts that for any fixed value of $\xi$, the distribution of $\tau$ is not altered by the circumstances of growth, so that for every $\xi$,

$$
\frac{h(\xi, \tau)}{f(\xi)} \equiv \frac{\mathscr{H}(\xi, \tau)}{\mathscr{C}(\tau)}
$$

where $\mathscr{C}(\tau)$, the frequency function of generation time $\tau$ of mothers of newly formed cells in exponentially growing populations, is given by

$$
\mathscr{C}(\tau)=2 \mathrm{e}^{-v \tau} \mathscr{P}(\tau),
$$


where $v$ is the specific growth rate of the population. Therefore, Powell argues that $\mathscr{P}(\tau)$ satisfies the integral equation

$$
\mathscr{P}(\tau)=\int_{0}^{\infty} 2 \mathrm{e}^{-v \xi} \mathscr{P}(\xi) \frac{h(\xi, \tau)}{f(\xi)} \mathrm{d} \xi
$$

Clearly, eqn (2) is true if eqn (I) is true, but it may be possible for eqn (2) to hold when eqn (I) does not. A third case, where neither eqn (I) nor eqn (2) holds, is discussed below.

\section{EXAMPLE}

\section{$A$ case where eqns (I) and (2) do not hold}

Marr, Painter \& Nilson (I969) outlined a stochastic model for the bacterial division cycle, based on the hypothesis of Cooper \& Helmstetter (I968). In this model, the initiation of a cycle of DNA synthesis is treated as a primary event where the waiting time between primary events is a random variable. Cell divisions are treated as secondary events that are coupled to the primary events with a second random variable defined as the division time. It is possible to derive expressions for the frequency functions of interdivision times, but these expressions are so complex that I have not been able to show from them that eqns (I) and (2) either hold or do not hold in my model. Therefore I consider a special case of the model. Assume that the waiting time between primary events is a constant that is necessarily equal to the doubling time $g$ of the population. Also assume that in one-half of the divisions the division time is a constant $c$ and in the remaining cases the division time is $c+d$. The delay in division, $d$, is assumed to occur at random.

In this example there are three possible generation times, i.e. $g-d, g$ and $g+d$. A cell with generation time $g-d$ must be formed with division time $c+d$ and must divide with division time $c$. A cell with generation time $g$ must either be formed and divide with division times both equal to $c$, or it must be formed and divide with division times both equal to $c+d$. Finally, a cell with generation time $g+d$ must be formed with division time $c$ and must divide with division time $c+d$. These four division patterns are presented in Fig. I for the special case where $c=0$. In the population where $f(\tau)$ is defined, each division pattern is equally likely, so that $f(\tau)$ is defined in this discrete case by the probabilities in Table I. A similar enumeration of all patterns of generation times of mothers and their daughters gives the joint probabilities $h(\xi, \tau)$ of Table 2 .

During exponential growth, the rate of occurrence, $p^{\prime}(t)$, of primary events at time $t$ must be

$$
p^{\prime}(t)=p^{\prime}(0) \mathrm{e}^{v t} \text {. }
$$

Furthermore, all cells formed at $t=0$ with generation times equal to $g-d$ must be the consequence of primary events occurring at time $-(c+d)$ since these cells must be formed with division time $c+d$. Thus the rate of formation of such cells is $\frac{1}{2} p^{\prime}(0) \mathrm{e}^{-v(c+d)}$. Similarly, the rate of formation at $t=0$ of cells with generation time $g$ is $\frac{1}{2} p^{\prime}(0)\left(\mathrm{e}^{-v c}+\mathrm{e}^{v(c+d)}\right)$, and the rate of formation at $t=0$ of cells with generation time $g+d$ is $\frac{1}{2} p^{\prime}(0) \mathrm{e}^{-v c}$. Normalization now gives the values of $\mathscr{P}(\tau)$ in Table $\mathrm{I}$.

In this discrete example, the integral in eqn (2) becomes a summation: if $\tau=g-d$, the summation is

$$
\sum_{\xi=g-d}^{\xi=g+d} 2 \mathrm{e}^{-v \xi} \mathscr{P}(\xi) \frac{h(\xi, \tau)}{f(\xi)}=\frac{\mathrm{I}+3 \mathrm{e}^{-v d}}{8+8 \mathrm{e}^{-v d}}
$$

Since this is not equal to $\mathscr{P}(g-d)$, eqn (2) does not hold in this case. Similarly, it is possible to show that eqn (I) also does not hold. 

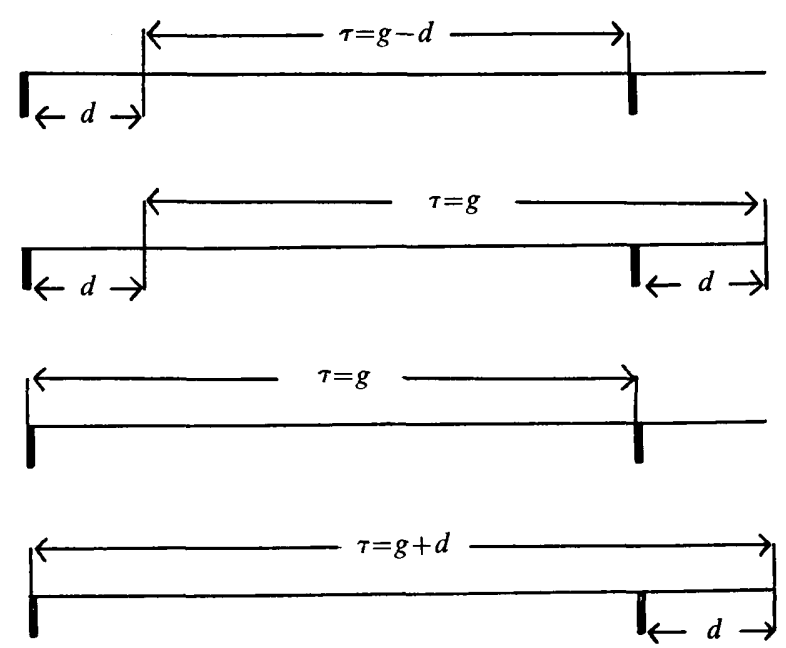

Fig. I. The four possible patterns of division in the example in the text. The long horizontal lines represent the time axis in each case. The heavy vertical lines indicate the occurrence of primary events in the cell cycle, and the thin vertical lines indicate cell divisions. Delays are indicated below the time axis and the interdivision times are indicated above. The interval between primary events is equal to $g$ in all cases.

Table I. The probabilities $f(\tau)$ and $\mathscr{P}(\tau)$, for the values of $\tau$ that are possible in the example in the text

$\begin{array}{ccc}\tau & \mathscr{P}(\tau) & f(\tau) \\ g-d & \frac{1}{2} \mathrm{e}^{-v d} /\left(\mathrm{I}+\mathrm{e}^{-v d}\right) & \frac{1}{4} \\ g & \frac{1}{2} & \frac{1}{2} \\ g+d & \frac{1}{2} \mathrm{e}^{-v d} /\left(\mathrm{I}+\mathrm{e}^{-v d}\right) & \frac{1}{4}\end{array}$

Table 2. Joint probability $h(\xi, \tau),{ }^{*}$ for mother-daughter pairs selected at random from the stationary process defined in the text

\begin{tabular}{|c|c|c|c|}
\hline \multirow{2}{*}{$\begin{array}{l}\text { Interdivision } \\
\text { time }(\xi)\end{array}$} & \multicolumn{3}{|c|}{$h(\xi, \tau)$, for $\tau$ equal to: } \\
\hline & $g-d$ & $g$ & $g+d$ \\
\hline$g-d$ & 0 & $\mathrm{I} / 8$ & $1 / 8$ \\
\hline$g$ & $\mathrm{I} / 8$ & $\mathrm{I} / 4$ & $I / 8$ \\
\hline$g+d$ & $1 / 8$ & $1 / 8$ & 0 \\
\hline
\end{tabular}

* The probability that a mother cell has interdivision time $\xi$ and the daughter interdivision time $\tau$.

\section{DISCUSSION}

The above example can be viewed as a special case of Powell's (1964) analysis of the Koch \& Schaechter hypothesis (if we assume equality of size of sisters at division) as well as a special case of my analysis of the Cooper \& Helmstetter (I968) hypothesis. The delay in division can also be interpreted as an experimental error in determining the time of separation of sister cells. Therefore, the example suggests that eqns (I) and (2) may not be totally consistent with data from the growth of microbial populations.

Equations ( 1 ) and (2) can hold in models where the generation times of mothers and their daughters are correlated: for example, in a model where the generation times in every line of descent alternate between $g-d$ and $g+d$, it can be shown that both eqn (I) and eqn (2) 
hold. I do not understand why they should hold in some cases and not in others and suggest that eqn (I) or (2) should be verified in any model where they are used.

Any difference between the 'real' and the 'artificial' distributions is relevant to the determination of interdivision times from the growth of synchronous cultures derived from membrane cultures (Helmstetter \& Cummings, 1964). In an ideal membrane culture, one of the two sister cells formed by each division is removed from the culture while the other remains on the membrane. Thus it is reasonable to treat growth of such cultures as a stationary process and to assume that the distribution of generation times in such cultures approaches the 'artificial' distribution. Therefore, one can be assured of measuring the 'real' distribution only by starting with cells shed from the membrane culture immediately after it is inoculated with cells from an exponentially growing population. However, in practice, this is not feasible since cells of all ages are shed at the inception of the culture. Marr et al. (1969) and Harvey (1972) have calculated the distribution of generation times of cells shed some time after the inception of the membrane culture and have presumably determined a transient function that is between the 'real' and the 'artificial' distributions.

If the magnitude of the delay $d$ is small compared with $g$, the fractional difference between $f(g \pm d)$ and $\mathscr{P}(g \pm d)$ is approximately $\frac{1}{2} \ln 2(g / d)$ in the example in the previous section. Thus the difference between $f$ and $\mathscr{P}$ may be negligible if the variance in delays is small enough. The variance in delays should be less than $\frac{1}{2} \sigma^{2}$, the variance in interdivision time (Marr et al. 1969). In the above example the variance in delays is $d^{2} / 4$. Therefore, $\frac{1}{2} \ln 2(d / g)$ should be less than $2^{-\frac{1}{2}} \ln 2(\sigma / g)$, so that the difference between $f(\tau)$ and $\mathscr{P}(\tau)$ at $2^{\frac{1}{2}}$ standard deviations from the mean of $f(\tau)$ is approximately $\frac{1}{2} \sigma / g$.

The example considered above is extreme in that all the variance in generation times results from delays in division. In less extreme models, the difference between $f$ and $\mathscr{P}$ may be much smaller. The above example does, however, show that if the coefficient of variation $\sigma / g$ is large, the difference between $f$ and $\mathscr{P}$ may be noticeable. In the case of Escherichia coli $\mathrm{B} / \mathrm{r}$, grown on membrane cultures (Marr et al. 1969), the coefficient of variation is approximately $0 \cdot 2$, so that the difference between $f$ and $\mathscr{P}$ should be small.

\section{REFERENCES}

Cooper, S. \& Helmstetter, C. (I968). Chromosome replication and the division cycle of Escherichia coli B/r. Journal of Molecular Biology 3I, 5I9-540.

HARveY, J. D. (1972). Parameters for the generation time distribution of Escherichia coli $\mathbf{B} / \mathrm{r}$. Journal of General Microbiology 70, I09-I I4.

Helmstetter, C. E. \& Cummings, D. J. (1964). An improved method for the selection of bacterial cells at division. Biochimica et biophysica acta 82, 608-6Io.

Marr, A. G., Painter, P. R. \& Nilson, E. H. (1969). Growth and division of individual bacteria. Microbial growth. Symposia of the Society for General Microbiology 19, 237-26r.

Powell, E. O. (1964). A note on Koch \& Schaechter's hypothesis about growth and fission of bacteria. Journal of General Microbiology 37, 23 I-249.

Powell, E. O. (1969). Generation times of bacteria: real and artificial distributions. Journal of General Microbiology 58, I4 I-I 44. 